\title{
Laterality in the perception of temporal cues of musical timbre
}

\author{
Alfredo Brancucci, Pietro San Martini* \\ Department of Psychology, First University of Rome, 'La Sapienza', Rome, Italy
}

Received 29 June 1998; received in revised form 29 January 1999; accepted 20 April 1999

\begin{abstract}
Laterality in the perception of non-stationary aspects of musical timbre was investigated in 54 right-handed non-musicians. Timbre differences were produced by altering the amplitude envelope of a steady-state complex tone. Two single-choice tests with attention directed to one ear were used-a dichotic test and a monaural test with contralateral white noise. Dependent variables were reaction time and accuracy. Both tests showed a significant left ear advantage for reaction time. For the accuracy variable, a significant left ear advantage was found only in the monaural test. Results are briefly discussed in terms of their compatibility with the generally accepted notion that spectral and temporal integrations of sounds are primarily functions of the right and left hemisphere, respectively. (C) 1999 Elsevier Science Ltd. All rights reserved.
\end{abstract}

Keywords: Hemispheric asymmetries; Dichotic listening; Monaural asymmetries; Complex tones

\section{Introduction}

While pitch and loudness of sounds have relatively well-understood relations to their underlying physical correlates, the psychoacoustical substrate of timbrebroadly defined as "that attribute of auditory sensation in terms of which a listener can judge that two sounds similarly presented and having the same loudness and pitch are dissimilar" (definition of the American Standards Association, cited in [18], p. 246) - is far more complex.

Since the 1860s, when Von Helmholtz carried out various experiments showing the dependence of timbre on the amplitude pattern of the harmonic spectrum [37], it has generally been recognised that musical timbre is associated with the spectral composition of tones (see [22] for a review). However, a definition of timbre solely in terms of spectral composition is strictly appropriate only with reference to steady state sounds since it leaves out those aspects of timbre perception that depend on temporal parameters such as the

\footnotetext{
* Corresponding author. Via dei Marsi 78, 00185 Rome, Italy. Tel.: + 39-6-4991-7623; fax: + 39-6-445-1667.

E-mail address: smartini@axrma.uniromal.it (P. San Martini)
}

characteristics of the attack and the rapid fluctuations of the amplitude or even of the spectral composition of the sound $[2,3,8,24,31,38]$. Reference to these parameters, for instance, explains why it is generally easy to recognise a certain musical timbre even through the distorted spectral output of a transistor radio or why it is difficult to recognise the timbre of a piano if it is reproduced backwards even though the original and the reverse sound have the same harmonic composition [3].

Investigations of lateral ear asymmetries for timbre perception using dichotic stimulation procedures have shown that a left ear advantage, indicative of a right hemisphere advantage, can be demonstrated for tasks involving 'natural' musical sounds, that is, sounds (generated by different musical instruments) that vary both in harmonic composition and in the temporal features of the amplitude envelope. This is in line with the general notion of right hemisphere dominance in the perception of musical sounds $[1,17,34,39,41]$. This left ear advantage, however, lacks robustness, as the following brief summary of the literature will show. Kallman and Corballis [13], by using a dichotic monitoring procedure and reaction time as a dependent variable, found a left ear advantage that disappeared, 
however, after the first block of 36 trials. In a later study devoted to expectancy effects in the determination of ear asymmetries, Kallman [12] found the expected right ear advantage for verbal stimuli, but was not able to confirm the previously found left ear advantage for timbre perception. Using a dichotic monitoring procedure, Prior and Troup [20] found faster response times for the left ear, but no asymmetry in accuracy for the perception of rhythm. No asymmetry for either response time or accuracy was found for the perception of timbre. More recently, by using a dichotic monitoring procedure and the number of correct responses as the dependent variable, Boucher and Bryden [4] found a left ear advantage for melodies but no advantage for timbre perception. Of relevance in this context is also a study by Rastatter and Gallaher [21], who found a left ear advantage for reaction time in a test requiring participants to judge whether two monaural tones of the same pitch presented in succession were played by the same or different instruments. The opinion of the present authors is that the inconsistency of results may, at least in part, be accounted for by the following two considerations.

Firstly, by using sounds produced by natural musical instruments, the aforementioned investigations allowed the laterality effect to be masked by an advantage in the opposite direction, possibly introduced as a result of verbal labelling of the stimuli. Indirect evidence for this effect comes from Tramo and Gazzaniga [35], whose study of split-brain patients found a left hemisphere advantage for the identification of common musical instruments. Secondly, the investigations failed to distinguish between the stationary and temporal aspects of timbre perception and it could be argued that reliance on temporal vs stationary cues may diminish or even reverse the laterality effects, since it is generally accepted that the left hemisphere is better at discriminating fine temporal events (see [19] for a review).

One way to further clarify the conditions that affect perceptual laterality for timbre perception could be to use synthetic sounds, which are less likely to be linked to specific labels, and to separately investigate the effects of the frequency composition of the sounds and those of its temporal features. In a previous study, one of the present authors [29] used artificial steady-state sounds of constant pitch that varied only in harmonic composition and found a clear-cut left ear advantage for the perception of timbre. This result was in accordance with Milner [17], who investigated the effect of temporal lobe removal in a timbre discrimination task based on spectral cues, and with Tramo and Gazzaniga [35], who showed that the right hemisphere of commissurotomised patients was superior to the left on the same type of task.

As a continuation, this study aims to assess whether a left ear advantage (indicative of right hemisphere dominance) can still be found when the task requires timbre discriminations based on temporal rather than spectral cues. To this end, we used timbral differences conveyed by rapid amplitude changes of tones of identical pitch and harmonic composition, and two laterality tests - a traditional dichotic test and a monaural test with contralateral white noise. The rationale for using two tests instead of one was simply to have a better chance of detecting a laterality effect if there was one. The monaural test was chosen because it was felt that, since our stimuli were likely to sound rather unnatural to most subjects, the task of disentangling regular dichotic pairs could be perceived as discouraging or disorienting and could prove to be too difficult, thus limiting the measuring power of the test. The choice of a monaural test as a measure of laterality is based on an extensive review of the literature on monaural studies by Bradshaw and Nettelton [5], which shows that hemispheric asymmetries can also be detected using monaural stimulations. According to this review, the traditional dichotic procedure is not necessary for the detection of hemispheric asymmetries and the concept of an ipsilateral occlusion mechanism produced by the dichotic competition of stimuli, as posited by Kimura [15], is not required to explain the genesis of ear advantages.

\section{Methods}

\subsection{Subjects}

Fifty-four subjects (38 females and 16 males) aged between 20 and 58 years (mean age $=26.04$, s.d. $=8.44)$, volunteered to take part in the experiment. They all declared they were right-handed and this was borne out by a hand preference questionnaire [25] $($ mean $=69.3$, s.d. $=35.1)$. None of them complained of any auditory impairment (no audiometric test was done). All were non-musicians.

\subsection{Stimuli}

The stimuli were synthesised on a PC Pentium 133 with audiocard Sound Blaster AWE 32 using the CSound language [36] for sound synthesis. The following procedure was used in order to obtain three sounds with identical frequency composition and different amplitude envelopes. First, three complex tones with an identical frequency spectrum, pitch and intensity were generated on the basis of the first eight harmonics of $500 \mathrm{~Hz}$. Then, their amplitude envelopes were differently modulated keeping total and peak amplitude identical between the tones. Power spectra and ampli- 


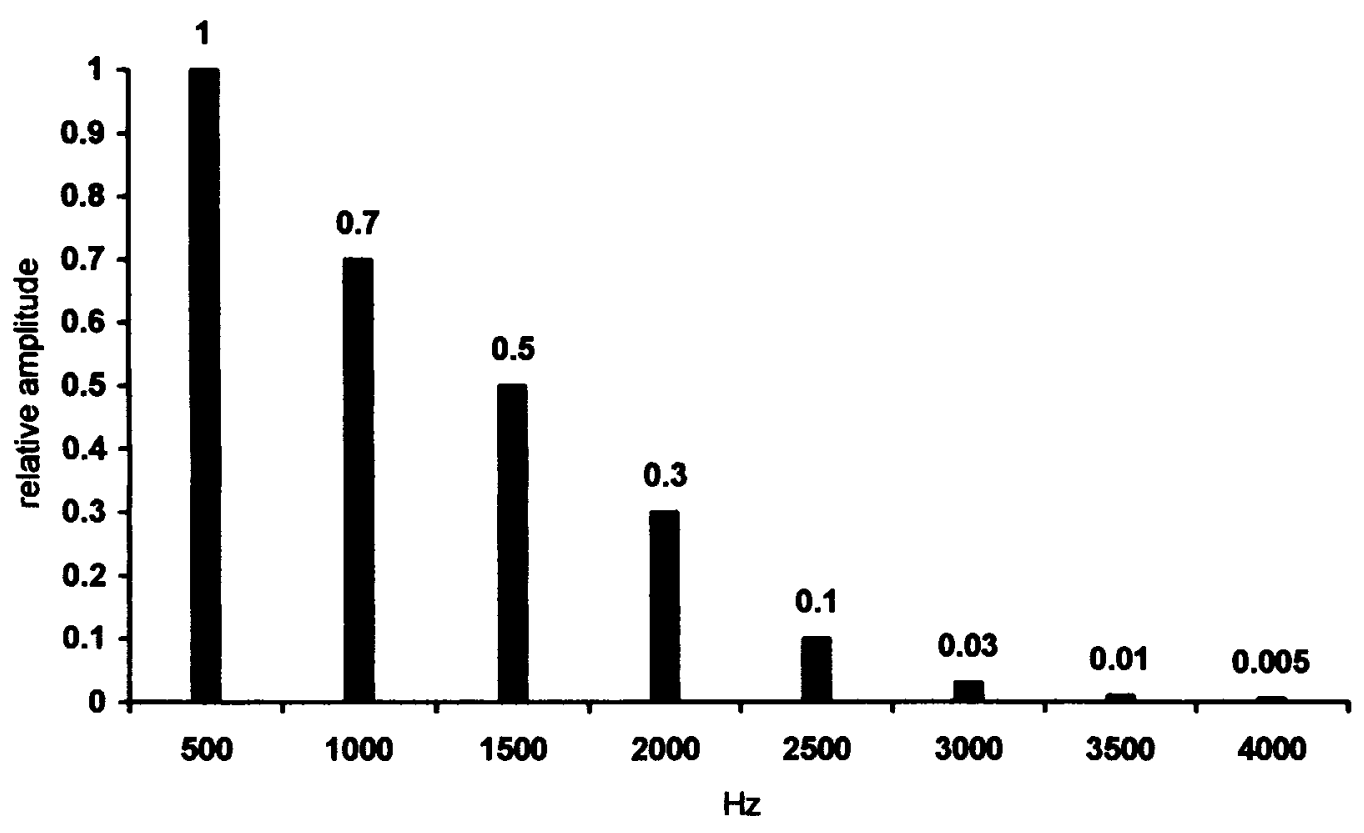

Fig. 1. Frequency spectrum of the stimuli of both tests.

tude envelopes of the stimuli are shown in Figs. 1 and 2.

Sampling rate was $22.05 \mathrm{kHz}$ and amplitude resolution 16 bit. To ensure that no transients or undesired alterations were present in the stimuli, they were recorded using headphones and re-analysed visually.

\subsection{The tests}

Subjects were presented with two tests - a dichotic one and a monaural one with contralateral white noise-both involving single choice and attention directed to one ear. This format was chosen because previous studies with musical material had shown that it allowed the detection of a consistent and reliable laterality effect even in musically uneducated subjects $[28,29,30]$.

\subsubsection{The dichotic test}

This was composed of 96 items with a $2 \mathrm{~s}$ inter-item interval. Each item consisted of the following sequence: one monaural tone $(500 \mathrm{~ms})$ followed by a pause $(1 \mathrm{~s})$, followed by a dichotic pair of completely aligned tones $(500 \mathrm{~ms})$. Half of the pairs had one sound which matched the probe, while the other half had no sounds which matched the probe. In case of matching, the matching tone was presented to the ear that had just received the probe.

\subsubsection{The monaural test}

This was also composed of 96 items with a $2 \mathrm{~s}$ interitem interval. Each item consisted of the following sequence: one monaural tone $(500 \mathrm{~ms})$ followed by a pause (1 s), followed by a pair consisting of one monaural tone and a contralateral burst of white noise (500 ms) beginning $50 \mathrm{~ms}$ before the contralateral tone and ending $50 \mathrm{~ms}$ after it, so that the tone was delivered when the noise was in its steady state.

Half of the pairs matched the probe. The amplitude shifts of the tones in this test, as compared to the dichotic test, were reduced in order to have a comparable degree of difficulty since preliminary trials had shown the contralateral white noise to have a lower masking effect with respect to contralateral tones (see Fig. 2).

In both tests the 96 items were grouped into 16 blocks of six items each. The side of presentation of the probe stimuli changed with every block. The blocks were separated by a $4 \mathrm{~s}$ interval. Each block was preceded by a beep $(200 \mathrm{~ms})$ which was presented monaurally to the ear that was to receive the subsequent probe stimulus of that block. Subjects were instructed to direct their attention, during the subsequent block, to the ear receiving the monaural beep and were informed that no intrusions were to be expected in the unattended ear.

\subsection{Procedure}

The dichotic and monaural tests were administered separately in two sessions. The order of test administration was counterbalanced across subjects and the second session took place within two weeks of the first. The experiment was completely automated by means of a Quick-Basic program. The procedure was identical for both tests. Subjects wore a pair of head- 


\section{Dichotic test}
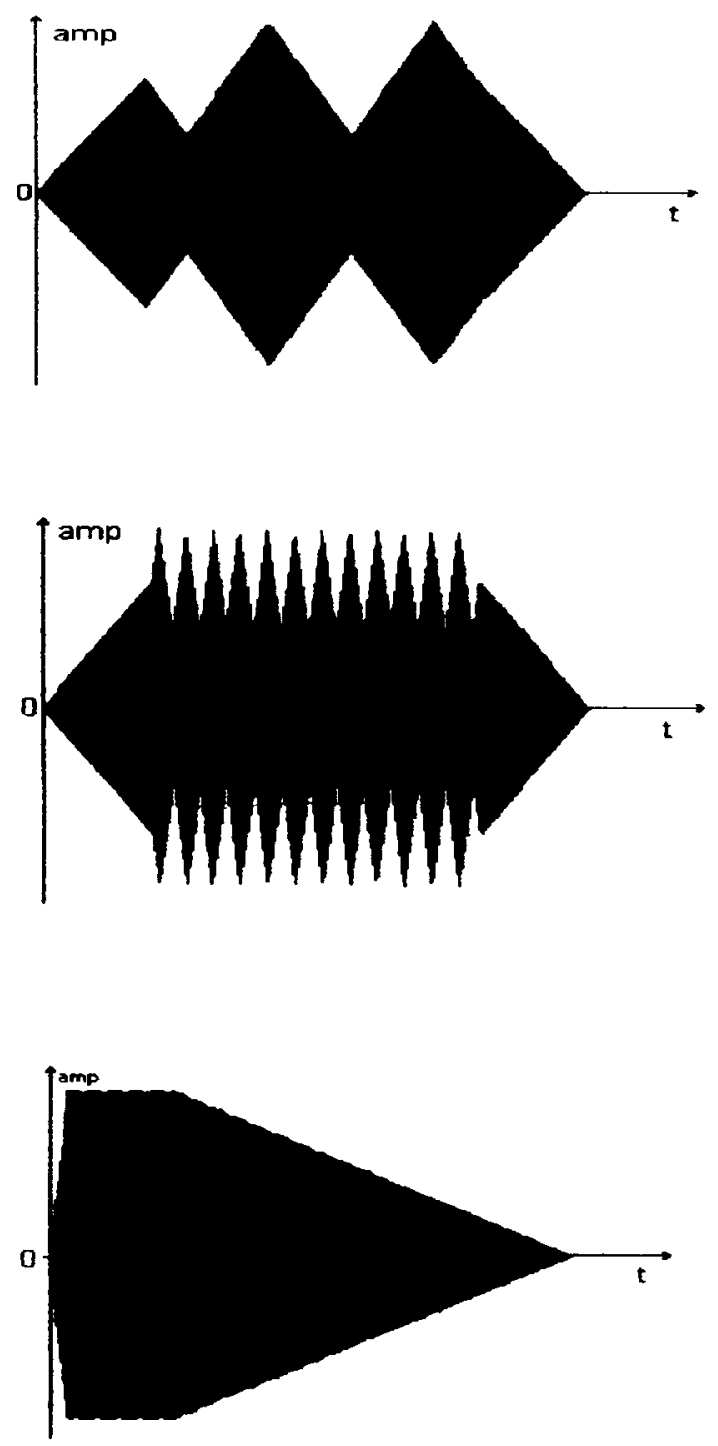

\section{Monaural test}
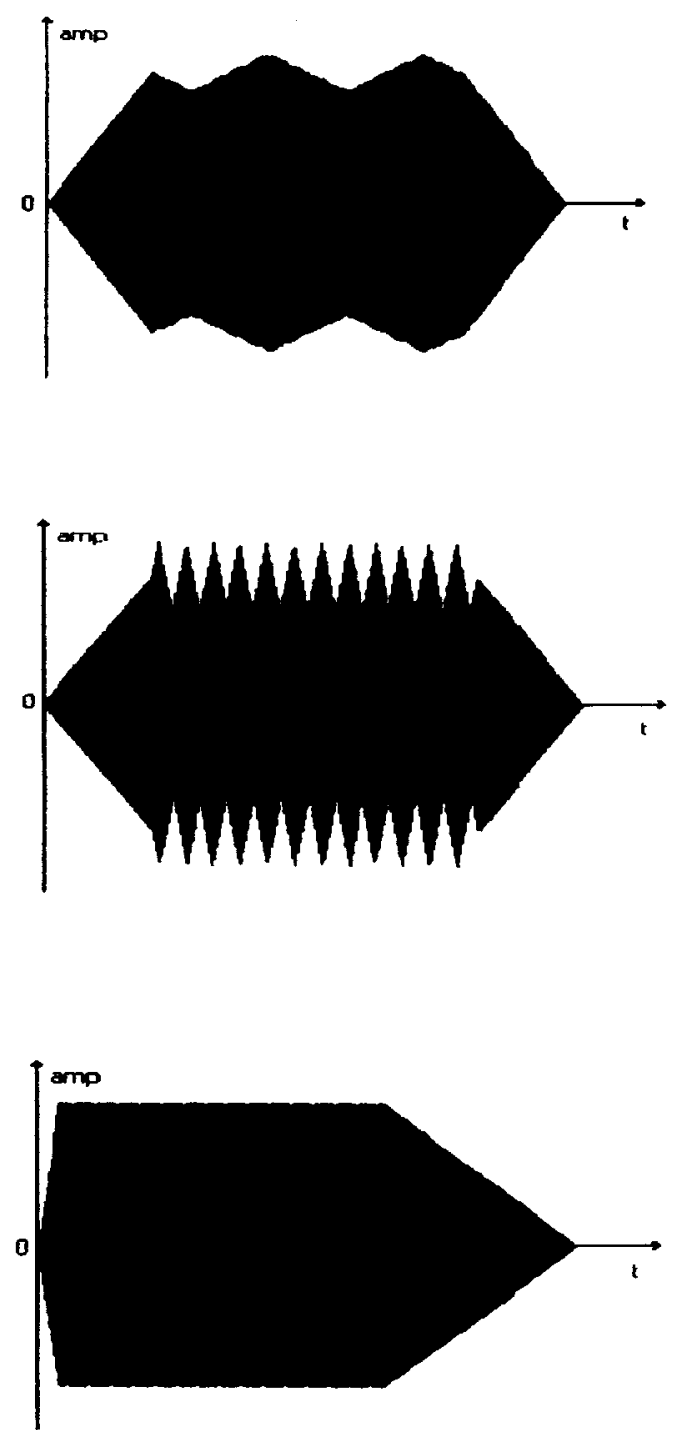

Fig. 2. Amplitude envelopes of the stimuli. Duration: $500 \mathrm{msec}$; peak amplitude: $78 \mathrm{dBA}$.

phones (AKG K141 Monitor, impedance 600 ohms) and were comfortably seated at a table in front of a computer monitor (approximately $70 \mathrm{~cm}$ from the subject's head) with both their hands lying on the keyboard. They were instructed to look at a green circle in the centre of the screen in front of them and not to shift their gaze laterally if possible during the experiment. In a first familiarisation phase, subjects were invited to listen to the sounds that were to be used in the subsequent test until they felt familiar with them. In the experimental phase subjects were presented with the test twice, the second time with the headphones rotated between the ears. The initial orientation of the headphones was counterbalanced across subjects. The level of the sounds was identical in both earphones, 78 dBA intensity as measured by a phonometer. Subjects had to judge whether the pair of sounds they had just heard contained the probe, by pressing one of two keys on the keyboard as fast as possible. They were told to press the ' $\mathrm{v}$ ' key (in case of a match) with their left hand forefinger or the ' $n$ ' key (in case of a mismatch) with their right hand forefinger. The associ- 

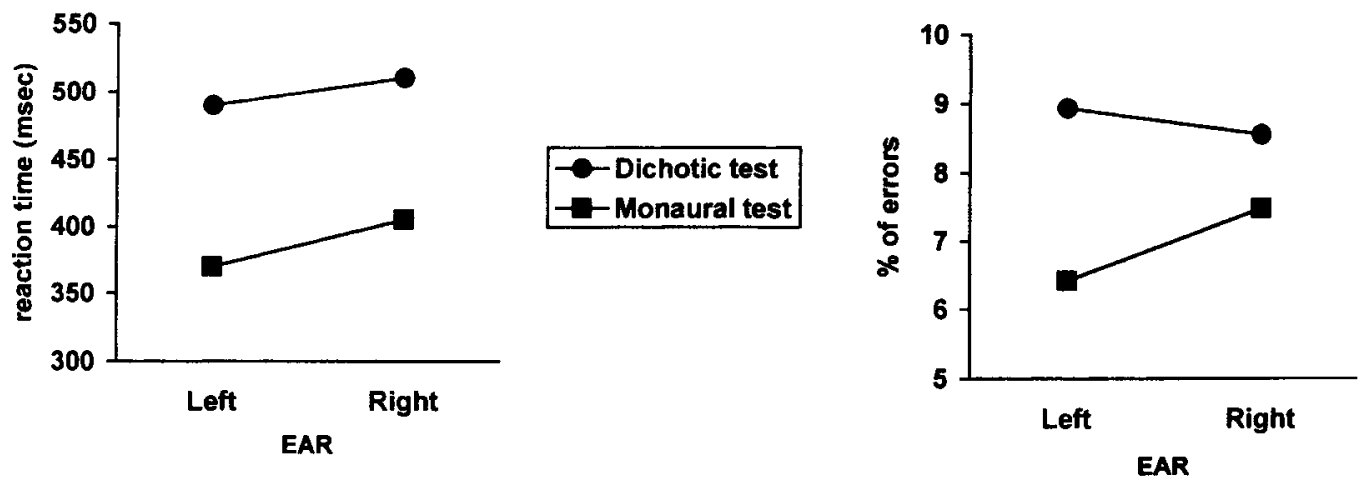

Fig. 3. Mean values of (median) reaction time and percent errors as a function of ear of presentation for the dichotic and monaural test.

ation between hand (left hand vs right hand) and type of response (yes vs no) was not counterbalanced. Type and latency of response were automatically stored for later analysis. Each experimental session lasted approximately $30 \mathrm{~min}$.

\section{Results}

The dependent variables were reaction time and number of errors. Reaction time was measured as the median latency of correct responses (in ms). The group mean values for both variables are depicted graphically as a function of ear of presentation in Fig. 3 .

In order to obtain normality and homogeneity of distributions of reaction time data, as they tended to be positively skewed, a logarithmic transformation was performed as suggested by the method described by Kirk ([16], p. 66-67). The same method suggested using untransformed scores for the number of errors.

Preliminary analyses of variance indicated that earphone position, the order of test administration (whether subjects first received the dichotic or the monaural test) and the sex of the subjects, did not influence the dependent variables as they showed no main or interaction effects. These variables were therefore not included in subsequent analyses.

A $2 \times 2$ repeated measure ANOVA with ear of input and type of test as independent variables was carried out for both dependent variables

As regards the reaction time variable, the ANOVA showed a significant main effect for the type of test $\left(F_{1,53}=44.14 ; P<0.001\right)$, due to a shorter reaction time in the monaural test, a significant main effect for ear of input $\left(F_{1,53}=11.49 ; P=0.001\right)$, due to a left ear advantage, and no interaction of ear by test $\left(F_{1,53}=1.06 ; P=0.308\right)$. According to Cohen's classification system ([9], p. 48-49), the effect size of the ear factor was between small and medium for the dichotic test $(d=0.37)$ and just above medium for the monaural test $(d=0.52)$.
For the number of errors, the ANOVA showed a significant main effect for the type of test $\left(F_{1,53}=9.24\right.$; $P=0.004)$, due to a smaller number of errors in the monaural test, no main effect for ear $\left(F_{1,53}=1.00\right.$; $P=0.321)$ and a significant interaction of type of test by ear $\left(F_{1,53}=9.71 ; P=0.003\right)$. A subsequent analysis of the simple main effects showed that there was a significant left ear advantage for the monaural test $\left(F_{1,53}=7.31 ; P=0.009\right)$ but not for the dichotic test $\left(F_{1,53}=0.74 ; p=0.394\right)$. Cohen's $d$ for the ear effect in the monaural test was just above medium $(d=0.54)$.

\section{Discussion}

Our results show that both a dichotic test and a monaural test requiring sound discrimination on the basis of timbral cues conveyed by rapid amplitude changes can produce a left ear advantage. This left ear advantage was consistent across reaction time and accuracy when contralateral white noise, rather than regular dichotic pairs, was used. With regular dichotic pairs, a significant ear advantage was obtained only with reaction time and it was of a smaller size. This result is similar to the one obtained by Kallman and Corballis [13], who found a laterality effect when reaction time, but not accuracy, was the dependent variable. This discrepancy between the two tests may perhaps be accounted for by their different levels of difficulty, since our monaural task was characterised by shorter reaction times and a higher accuracy. This interpretation, however, does not explain why the degree of difficulty affected the ear advantage of our subjects only as regards accuracy and not reaction time.

On the whole, our main finding of a left ear advantage for timbral qualities conveyed by temporal cues is in accordance with a report by Samson and Zatorre [28] on the effect of cerebral lesions on the ability to discriminate timbres differing in temporal components. These authors used two timbre discrimination tasks, 
one based on spectral cues (variation in the number of harmonics) and one on temporal cues (variation in the duration of the attack). Contrary to their own expectations, they found that right temporal patients performed significantly worse than left temporal and control patients, not only when the task was based on spectral cues, but also when the task was based on temporal cues. Our results seem to further support their conclusion that in both cases the features of sound, which are important for timbre discrimination, are mainly processed in the right temporal lobe of the human brain.

At first sight, this finding would seem to contradict the generally accepted dichotomy according to which, whilst the harmonic integration of sounds is primarily a function of the right hemisphere [7,23,26,29,35,40], the temporal integration of auditory events mainly involves left hemispheric structures [6,10,11,14,32,33].

It should be considered, however, that temporal and spectral cues of timbre perception interact at a physical level. Changes of the amplitude envelope of an otherwise constant sound tend to introduce inharmonic components generally confined to the lower frequency regions. It may be hypothesised that these components, although of low amplitude, play a decisive role in the perception of the temporal aspects of timbre and that rapid changes of the temporal amplitude envelope of sound that are relevant for timbre perception are in fact processed in the frequency, rather than time, domain. Should this be the case, an advantage of the left ear would be expected, even maintaining the dichotomy frequency domain-right hemisphere vs time domain-left hemisphere.

Along similar lines, it might be argued that left ear advantage occured in our subjects on the basis of an analysis, in the frequency domain, of the (inharmonic) spectral cues that were surreptitiously introduced by varying the amplitude envelope of the steady state signals, even though a simultaneous analysis of the amplitude variations in the time domain tended to produce a laterality effect in the opposite direction or no laterality effect at all. In other words, it may be argued that the ear advantage shown by our subjects was the net effect of two factors operating in opposite directions and leading cumulatively to a moderate left ear advantage. However, to say something definite on the contribution of non-stationary cues to cerebral asymmetries in timbre discrimination, what is ideally needed is a single experiment requiring subjects to make two types of timbre discriminations, one based on stationary tones and one on non-stationary ones, with both tasks of the same difficulty. An interaction between ear and type of stimulus, with significantly smaller or reversed (left) ear advantage for non-stationary stimuli, would then indicate that there is a dissociation between the two processing modes and the hemi- spheres; or it would at least show that there is less dominance of the right hemisphere in analysing complex tones in the time domain than in the frequency domain. Our hypothesis - that left ear advantage for temporal cues of timbre perception is based on a frequency analysis of residual spectral components introduced by amplitude variations - might gain further support if future investigations can demonstrate that left ear advantage for non-stationary tones occurs only when amplitude variations are so rapid that they are perceived holistically, as timbral qualities, and also that left ear advantage reverses (or disappears) when amplitude variations are perceived as changes of loudness in time.

\section{Acknowledgements}

This paper is partially based on a thesis by the first author, which was submitted to Rome's 'La Sapienza' University in July 1997. This research was supported by grants to P. San Martini from the Ministero per l'Università e la Ricerca Scientifica e Tecnologica.

\section{References}

[1] Auzou P, Eustache F, Etevenon P, Platel H, Rioux P, Lambert J, Lechevalier B, Zarifian E, Baron JC. Topographic EEG activations during timbre and pitch discrimination tasks using musical sounds. Neuropsychologia 1995;33:25-37.

[2] Balzano GJ. What are musical pitch and timbre? Music Perception 1986;3:297-314.

[3] Berger KW. Some factors in the recognition of timbre. Journal of the Acoustical Society of America 1964;36:1888-91.

[4] Boucher R, Bryden MP. Laterality effects in the processing of melody and timbre. Neuropsychologia 1997;35:1467-73.

[5] Bradshaw JL, Nettelton NC. Monaural asymmetries. In: Hugdahl K, editor. Handbook of dichotic listening: theory, methods and research. New York: Wiley and Sons, 1988. p. 4570.

[6] Carmon A, Nachshon I. Effect of unilateral brain damage on perception of temporal order. Cortex 1981;7:410-8.

[7] Chobor KL, Brown JW. Phoneme and timbre monitoring in left and right cerebrovascular accident patients. Brain and Language 1987;30:278-84.

[8] Clark M, Robertson JP, Luce D. A preliminary experiment on the perceptual basis for musical instrument families. J Audio Engng Soc 1964;12:199-203.

[9] Cohen J. Statistical power analysis for the behavioral sciences. New York: Academic Press, 1977.

[10] Efron R. Temporal perception, aphasia and déjà vu. Brain 1963;86:403-24.

[11] Gordon HW. Left hemisphere dominance for rhythmic elements in dichotically-presented melodies. Cortex 1978;14:58-70.

[12] Kallman HJ. Can expectancy explain reaction time ear asymmetries? Neuropsychologia 1978;16:225-8.

[13] Kallman HJ, Corballis MC. Ear asymmetry in reaction time to musical sounds. Perception \& Psychophysics 1975;17:368-70.

[14] Kester DB, Saykin AJ, Sperling MR, O'Connor MJ, Robinson 
LJ, Gur RC. Acute effect of anterior temporal lobectomy on musical processing. Neuropsychologia 1991;29:703-8.

[15] Kimura D. Functional asymmetry of the brain in dichotic listening. Cortex 1967;3:163-8.

[16] Kirk RE. Experimental design: procedures for the behavioral sciences. Belmont CA: Brooks/Cole Publishing Company, 1968.

[17] Milner B. Laterality effects in audition. In: Mountcastle VB, editor. Interhemispheric relations and cerebral dominance. Baltimore: John Hopkins Press, 1962. p. 177-95.

[18] Moore BCJ. An introduction to the psychology of hearing, 4th ed. London: Academic Press, 1997.

[19] Nicholls MER. Temporal processing asymmetries between the cerebral hemispheres: evidence and implications. Laterality 1996;1:97-138.

[20] Prior M, Troup GA. Processing of timbre and rhythms in musicians and non-musicians. Cortex 1988;24:451-6.

[21] Rastatter MP, Gallaher AJ. Reaction times of normal subjects to monaurally presented verbal and tonal stimuli. Neuropsychologia 1982;20:465-73.

[22] Risset J-C, Wessel DL. Exploration of timbre by analysis and synthesis. In: Deutsch D, editor. The psychology of music. New York: Academic Press, 1982. p. 25-58.

[23] Robin DA, Tranel D, Damasio H. Auditory perception of temporal and spectral events in patients with focal left and right cerebral lesions. Brain and Language 1990;39:539-55.

[24] Saldanha EL, Corso JF. Timbre cues and the identification of musical instruments. Journal of the Acoustical Society of America 1964;36:2021-6.

[25] Salmaso D, Longoni AM. Problems in assessment of hand preference. Cortex 1985;21:533-49.

[26] Samson S, Zatorre RJ. Melodic and harmonic discrimination following unilateral cerebral excision. Brain and Cognition 1988;7:348-60.

[27] Samson S, Zatorre RJ. Contribution of the right temporal lobe to musical timbre discrimination. Neuropsychologia 1994;32:231-40.

[28] San Martini P, De Gennaro L, Filetti F, Lombardo C, Violani C. Prevalent direction of reflective lateral eye movements and ear asymmetries in a dichotic test of musical chords. Neuropsychologia 1994;32:1515-22.
[29] San Martini P, Filetti F, Marangon L, Tasin N. Asimmetrie emisferiche nella percezione del timbro: vantaggio dicotico per toni stazionari sintetici di diversa composizione spettrale. In: XIII Congresso Nazionale della divisione ricerca di base in Psicologia (S.I.Ps.), Rivista di Psicologia, 1994. 1. p. 36-37.

[30] San Martini P, Quarta R. Dichotic advantage in the perception of musical chords: effects of memory and attentional load and stability of individual differences. Rassegna di Psicologia 1989;6:137-43.

[31] Schouten JF. The perception of timbre. In: Report of the 6th International Congress on Acoustics, GP6-2, 1968.

[32] Swisher L, Hirsch IJ. Brain damage and the ordering of two temporally successive stimuli. Neuropsychologia 1972;10:13751.

[33] Tallal P, Newcombe F. Impairment of auditory perception and language comprehension in dysphasia. Brain and Language 1978;5:13-24

[34] Tramo MJ, Bharucha JJ. Musical priming by the right hemisphere post-callosotomy. Neuropsychologia 1991;29:313-25.

[35] Tramo MJ, Gazzaniga MS. Discrimination and recognition of complex tonal spectra by the cerebral hemispheres: differential lateralization of acoustic-discriminative and semantic associative functions in auditory pattern perception. Society of Neuroscience Abstracts 1989;15:421-2.

[36] Vercoe BL. A manual for the audio processing system and supporting programs with tutorials. Massachusetts Institute of Technology: Media Labs, 1992.

[37] Von Helmholtz H. Thèorie psychologique de la musique fondée sur l'étude des sensations auditives. Paris: Masson, 1869.

[38] Wedin L, Goude G. Dimension analysis of the perception of instrumental timbre. Scand J Psychol 1972;13:228-40.

[39] Zatorre RJ. Discrimination and recognition of tonal melodies after unilateral cerebral excision. Neuropsychologia 1985;23:3141.

[40] Zatorre RJ. Pitch perception of complex tones and human temporal-lobe function. Journal of the Acoustical Society of America 1988;84:566-72.

[41] Zatorre RJ, Evans AC, Meyer E. Functional activation of right temporal and occipital cortex in processing tonal melodies. Journal of the Acoustical Society of America 1993;93:2364. 